\title{
Existence results for impulsive fractional integro-differential equation of mixed type with constant coefficient and antiperiodic boundary conditions
}

\author{
Mingyue Zuo ${ }^{1}$, Xinan Hao ${ }^{1 *}$, Lishan Liu' ${ }^{1,2}$ and Yujun Cui ${ }^{3}$
}

\author{
*Correspondence: \\ haoxinan2004@163.com \\ 'School of Mathematical Sciences, \\ Qufu Normal University, Qufu, \\ Shandong 273165, P.R. China \\ Full list of author information is \\ available at the end of the article
}

\begin{abstract}
In this paper, we are concerned with the existence and uniqueness of solutions for impulsive fractional integro-differential equation of mixed type with constant coefficient and antiperiodic boundary condition. Our results are based on the Banach contraction mapping principle and the Krasnoselskii fixed point theorem. Some examples are also given to illustrate our results.
\end{abstract}

Keywords: antiperiodic boundary value problems; impulsive; fractional integro-differential equations; existence results

\section{Introduction}

Fractional differential equations appear naturally in a number of fields such as physics, chemistry, electromagnetic, engineering, control, and other branches; see [1-16] and the references therein. Fractional differential equations have recently gained much importance and attention. The study of fractional differential equations ranges from the theoretical aspects of the existence of solutions to the analytic and numerical methods for finding solutions.

Impulsive differential equations arising from the real world describe the dynamics of processes in which sudden discontinuous jumps occur. Such processes are naturally seen in physics, engineering, biology, and so on. Due to their significance, it is important to study the solvability of impulsive differential equations. Impulsive differential equations of fractional order have not been much studied, and many aspects of these equations are yet to be explored. The recent results on impulsive fractional differential equations can be found in [17-32] and the references therein.

Recently, the boundary value problem of impulsive fractional differential equations with antiperiodic boundary conditions have been studied in the literature; see [33-39]. The authors of [36-38] investigated the following antiperiodic boundary value problem for

(c) The Author(s) 2017. This article is distributed under the terms of the Creative Commons Attribution 4.0 International License (http://creativecommons.org/licenses/by/4.0/), which permits unrestricted use, distribution, and reproduction in any medium, provided you give appropriate credit to the original author(s) and the source, provide a link to the Creative Commons license, and indicate if changes were made. 
impulsive differential equations of fractional order:

$$
\left\{\begin{array}{l}
{ }^{\mathrm{c}} D^{q} u(t)=f(t, u(t)), \quad t \in[0, T] \backslash\left\{t_{1}, t_{2}, \ldots, t_{m}\right\}, 1<q \leq 2, \\
\left.\Delta u\right|_{t=t_{k}}=I_{k}\left(u\left(t_{k}\right)\right),\left.\quad \Delta u^{\prime}\right|_{t=t_{k}}=J_{k}\left(u\left(t_{k}\right)\right), \quad k=1,2, \ldots, m, \\
u(0)=-u(T), \quad u^{\prime}(0)=-u^{\prime}(T),
\end{array}\right.
$$

where ${ }^{\mathrm{c}} D^{q}$ is the Caputo fractional derivative of order $q, f:[0, T] \times \mathbf{R} \rightarrow \mathbf{R}$ is continuous, $I_{k}, J_{k}: \mathbf{R} \rightarrow \mathbf{R}, \mathbf{R}=(-\infty,+\infty), 0=t_{0}<t_{1}<\cdots<t_{m}<t_{m+1}=T$. By applying the Banach contraction mapping principle, Krasnoselskii fixed point theorem, Schaefer fixed point theorem, and a nonlinear alternative of the Leray-Schauder-type theorem, some existence results of solutions are obtained.

However, the existence and uniqueness of solutions to impulsive fractional differential equations for antiperiodic boundary value problems with constant coefficients seem to be rarely involved. It should be pointed out that Kilbas et al. (see (3.1.32)-(3.1.34) in [1]) obtained that the solution $u$ of the linear fractional differential equation with constant coefficients

$$
\left\{\begin{array}{l}
{ }^{\mathrm{c}} D^{q} u(t)+\lambda u(t)=h(t), \quad t \in[0,1], 0<q<1, \\
u(0)=u_{0}
\end{array}\right.
$$

is given by

$$
u(t)=E_{q}\left(-t^{q} \lambda\right) u_{0}+\int_{0}^{t}(t-s)^{q-1} E_{q, q}\left(-(t-s)^{q} \lambda\right) h(s) d s, \quad t \in[0,1]
$$

where $E_{q}$ and $E_{q, q}$ are the so-called classical and generalized Mittag-Leffler functions.

More recently, Wang and Lin [40] studied antiperiodic boundary value problems for impulsive fractional differential equations with constant coefficients

$$
\left\{\begin{array}{l}
{ }^{\mathrm{c}} D^{q} u(t)+\lambda u(t)=f(t, u(t)), \quad t \in J^{\prime}=J \backslash\left\{t_{1}, t_{2}, \ldots, t_{m}\right\}, 0<q<1, \\
\left.\Delta u\right|_{t=t_{k}}=u\left(t_{k}^{+}\right)-u\left(t_{k}^{-}\right)=y_{k}, \quad k=1,2, \ldots, m, \\
u(0)=-u(1),
\end{array}\right.
$$

where $\lambda>0, y_{k} \in \mathbf{R},{ }^{c} D^{q}$ is the Caputo fractional derivative of order $q \in(0,1), f: J \times \mathbf{R} \rightarrow \mathbf{R}$, $J=[0,1]$, and the fixed impulsive times $t_{k}$ satisfy $0=t_{0}<t_{1}<\cdots<t_{m}<t_{m+1}=1$. By means of fixed point theorems, some sufficient conditions on the existence and uniqueness of solutions for problem (1.2) are established under Lipschitz and nonlinear growth conditions.

Motivated by the works mentioned and many known results, in this paper, we are concerned with the existence and uniqueness of solutions for impulsive fractional integrodifferential equation of mixed type with constant coefficients and antiperiodic boundary condition

$$
\left\{\begin{array}{l}
{ }^{c} D^{q} u(t)+\lambda u(t)=f(t, u(t), T u(t), S u(t)), \quad t \in J^{\prime}=J \backslash\left\{t_{1}, t_{2}, \ldots, t_{m}\right\}, \\
\left.\Delta u\right|_{t=t_{k}}=I_{k}\left(u\left(t_{k}\right)\right), \quad k=1,2, \ldots, m, \\
u(0)=-u(1),
\end{array}\right.
$$


where ${ }^{\mathrm{c}} D^{q}$ is the Caputo fractional derivative of order $q \in(0,1), \lambda>0,0=t_{0}<t_{1}<\cdots<$ $t_{m}<t_{m+1}=1, f \in C(J \times \mathbf{R} \times \mathbf{R} \times \mathbf{R}, \mathbf{R}), J=[0,1], \mathbf{R}$ is the set of real numbers, $\left.\Delta u\right|_{t=t_{k}}$ denotes the jump of $u(t)$ at $t=t_{k}$, that is, $\left.\Delta u\right|_{t=t_{k}}=u\left(t_{k}^{+}\right)-u\left(t_{k}^{-}\right)$, where $u\left(t_{k}^{+}\right)$and $u\left(t_{k}^{-}\right)$represent the right and left limits of $u(t)$ at $t=t_{k}$, respectively, $T$ and $S$ are the linear operators defined by

$$
(T u)(t)=\int_{0}^{t} k(t, s) u(s) d s \quad \text { and } \quad(S u)(t)=\int_{0}^{1} h(t, s) u(s) d s, \quad t \in J,
$$

where $k \in C(D, \mathbf{R}), D=\{(t, s) \in J \times J: t \geq s\}$, and $h \in C(J \times J, \mathbf{R})$.

At present, the concept of solutions for impulsive fractional differential equations has been argued extensively. There are some ways to consider the notion of solution for impulsive fractional differential equations; for example, see [29-32]. In this paper, we adopt the formula of the solution in Lemma 2.4, which comes from [40].

This paper is arranged as follows. In Section 2, we present some definitions and preliminary lemmas. In Section 3, we establish the existence and uniqueness of solutions for the boundary value problem (1.3) by using the Banach contraction mapping principle and Krasnoselskii fixed point theorem. Some illustrated examples are presented in Section 4.

\section{Preliminaries and lemmas}

Let $J_{0}=\left[0, t_{1}\right], J_{1}=\left(t_{1}, t_{2}\right], \ldots, J_{m-1}=\left(t_{m-1}, t_{m}\right], J_{m}=\left(t_{m}, 1\right]$, and

$$
\begin{aligned}
P C(J, \mathbf{R})= & \left\{u: J \rightarrow \mathbf{R}: u \in C\left(J_{k}, \mathbf{R}\right), k=0,1,2, \ldots, m,\right. \\
& \left.u\left(t_{k}^{+}\right) \text {and } u\left(t_{k}^{-}\right) \text {exist }, k=1, \ldots, m \text {, and } u\left(t_{k}^{-}\right)=u\left(t_{k}\right)\right\} .
\end{aligned}
$$

Then $P C(J, \mathbf{R})$ is a Banach space with the norm $\|u\|_{P C}=\sup \{|u(t)|: t \in J\}$. For a measurable function $\mu: J \rightarrow \mathbf{R}$, define the norm

$$
\|\mu\|_{L^{p}(J)}= \begin{cases}\left(\int_{J}|\mu(t)|^{p} d t\right)^{\frac{1}{p}}, & 1 \leq p<\infty \\ \inf _{\operatorname{mes}(\bar{J})=0}\left\{\sup _{t \in J \bar{J}}|u(t)|\right\}, & p=\infty\end{cases}
$$

Then $L^{p}(J, \mathbf{R})$ is the Banach space of Lebesgue-measurable functions $\mu: J \rightarrow \mathbf{R}$ with $\|\mu\|_{L^{p}()}<\infty$.

Definition 2.1 ([1]) The fractional integral of order $\alpha$ with lower limit zero for a function $f:[0, \infty) \rightarrow \mathbf{R}$ is defined as

$$
I^{\alpha} f(t)=\frac{1}{\Gamma(\alpha)} \int_{0}^{t}(t-s)^{\alpha-1} f(s) d s,
$$

provided that the right-hand side is pointwise defined on $[0,+\infty)$.

Definition 2.2 ([1]) The Caputo derivative of order $\alpha$ for a function $f:[0, \infty) \rightarrow \mathbf{R}$ can be written as

$$
{ }^{\mathrm{c}} D^{\alpha} f(t)=\frac{1}{\Gamma(n-\alpha)}\left(\frac{d}{d t}\right)^{n} \int_{0}^{t} \frac{f(s)-\sum_{k=0}^{n-1} \frac{s^{k}}{k !} f^{(k)}(0)}{(t-s)^{\alpha+1-n}} d s, \quad t>0, n=[\alpha]+1,
$$

where $[\alpha]$ denotes the integer part of $\alpha$. 
Remark 2.1 ([30]) If $f \in C^{n}[0,+\infty)$, then

$$
{ }^{\mathrm{c}} D^{\alpha} f(t)=\frac{1}{\Gamma(n-\alpha)} \int_{0}^{t}(t-s)^{n-\alpha-1} f^{(n)}(s) d s=I^{n-\alpha} f^{(n)}(t), \quad t>0, n=[\alpha]+1,
$$

that is, Definition 2.2 is just the usual Caputo fractional derivative. In this paper, we consider an impulsive problem, so Definition 2.2 is appropriate.

Definition 2.3 A function $u \in P C(J, \mathbf{R})$ is said to be a solution of problem (1.3) if it satisfies the equation ${ }^{\mathrm{c}} D^{q} u(t)+\lambda u(t)=f(t, u(t), \operatorname{Su}(t), T u(t))$ a.e. on $J^{\prime}$ and the conditions $\left.\Delta u\right|_{t=t_{k}}=$ $I_{k}\left(u\left(t_{k}\right)\right), k=1, \ldots, m$, and $u(0)=-u(1)$.

Lemma 2.1 ([41]) The nonnegative functions $E_{q}$ and $E_{q, q}$ given by

$$
E_{q}(z)=\sum_{k=0}^{\infty} \frac{z^{k}}{\Gamma(q k+1)}, \quad E_{q, q}(z)=\sum_{k=0}^{\infty} \frac{z^{k}}{\Gamma(q k+q)},
$$

have the following properties:

(1) For any $\lambda>0$ and $t \in J$,

$$
E_{q}\left(-t^{q} \lambda\right) \leq 1, \quad E_{q, q}\left(-t^{q} \lambda\right) \leq \frac{1}{\Gamma(q)}
$$

Moreover,

$$
E_{q}(0)=1, \quad E_{q, q}(0)=\frac{1}{\Gamma(q)} .
$$

(2) For any $\lambda>0$ and $t_{1}, t_{2} \in J$,

$$
\begin{aligned}
& E_{q}\left(-t_{2}{ }^{q} \lambda\right) \rightarrow E_{q}\left(-t_{1}{ }^{q} \lambda\right) \quad \text { as } t_{2} \rightarrow t_{1}, \\
& E_{q, q}\left(-t_{2}{ }^{q} \lambda\right) \rightarrow E_{q, q}\left(-t_{1}{ }^{q} \lambda\right) \quad \text { as } t_{2} \rightarrow t_{1} .
\end{aligned}
$$

(3) For any $\lambda>0$ and $t_{1}, t_{2} \in J$ such that $t_{1} \leq t_{2}$,

$$
E_{q}\left(-t_{2}{ }^{q} \lambda\right) \leq E_{q}\left(-t_{1}{ }^{q} \lambda\right), \quad E_{q, q}\left(-t_{2}{ }^{q} \lambda\right) \leq E_{q, q}\left(-t_{1}{ }^{q} \lambda\right) .
$$

Lemma 2.2 ([42]) Let $M$ be a closed, convex, and nonempty subset of a Banach space $X$, and let $A, B$ be operators such that:

(1) $A x+B y \in M$ whenever $x, y \in M$.

(2) A is compact and continuous.

(3) B is a contraction mapping.

Then there exists $z \in M$ such that $z=A z+B z$.

Lemma 2.3 ([43]) Let $X$ be a Banach space, and let $J=[0, T]$. Suppose that $W \subset P C(J, X)$ satisfies the following conditions:

(1) $W$ is a uniformly bounded subset of $P C(J, X)$.

(2) $W$ is equicontinuous in $\left(t_{k}, t_{k+1}\right), k=0,1, \ldots, m$, where $t_{0}=0, t_{m+1}=T$. 
(3) Its $t$-sections $W(t)=\left\{u(t): u \in W, t \in J \backslash\left\{t_{1}, \ldots, t_{m}\right\}\right\}, W\left(t_{k}^{+}\right)=\left\{u\left(t_{k}^{+}\right): u \in W\right\}$, and $W\left(t_{k}^{-}\right)=\left\{u\left(t_{k}^{-}\right): u \in W\right\}$ are relatively compact subsets of $X$.

Then $W$ is a relatively compact subset of $P C(J, X)$.

Lemma 2.4 ([40]) Let $h: J \rightarrow \mathbf{R}$ be a continuous function. The function $u$ given by

$$
u(t)=\left\{\begin{array}{l}
\frac{-E_{q}(-\lambda) E_{q}\left(-t^{q} \lambda\right)}{1+E_{q}(-\lambda)} \sum_{i=1}^{m} \frac{y_{i}}{E_{q}\left(-t_{i}^{q} \lambda\right)}+\int_{0}^{t}(t-s)^{q-1} E_{q, q}\left(-(t-s)^{q} \lambda\right) h(s) d s \\
\quad-\frac{E_{q}\left(-t^{q} \lambda\right)}{1+E_{q}(-\lambda)} \int_{0}^{1}(1-s)^{q-1} E_{q, q}\left(-(1-s)^{q} \lambda\right) h(s) d s, \quad t \in J_{0}, \\
\frac{E_{q}\left(-t^{q} \lambda\right)}{1+E_{q}(-\lambda)}\left\{\sum_{i=1}^{m} \frac{y_{i}}{E_{q}\left(-t_{i}^{q} \lambda\right)}-\int_{0}^{1}(1-s)^{q-1} E_{q, q}\left(-(1-s)^{q} \lambda\right) h(s) d s\right\} \\
\quad-E_{q}\left(-t^{q} \lambda\right) \sum_{j=k+1}^{m} \frac{y_{j}}{E_{q}\left(-t_{j}^{q} \lambda\right)} \\
\quad+\int_{0}^{t}(t-s)^{q-1} E_{q, q}\left(-(t-s)^{q} \lambda\right) h(s) d s, \quad t \in J_{k}, k=1,2, \ldots, m-1, \\
\frac{E_{q}\left(-t^{q} \lambda\right)}{1+E_{q}(-\lambda)}\left\{\sum_{i=1}^{m} \frac{y_{i}}{E_{q}\left(-t_{i}^{q} \lambda\right)}-\int_{0}^{1}(1-s)^{q-1} E_{q, q}\left(-(1-s)^{q} \lambda\right) h(s) d s\right\} \\
\quad+\int_{0}^{t}(t-s)^{q-1} E_{q, q}\left(-(t-s)^{q} \lambda\right) h(s) d s, \quad t \in J_{m},
\end{array}\right.
$$

is a unique solution of the impulsive problem

$$
\left\{\begin{array}{l}
{ }^{\mathrm{c}} D^{q} u(t)+\lambda u(t)=h(t), \quad t \in J^{\prime} \\
\left.\Delta u\right|_{t=t_{k}}=y_{k}, \quad k=1,2, \ldots, m, \\
u(0)=-u(1) .
\end{array}\right.
$$

It follows from Lemma 2.4 that the solution of (1.3) can be expressed by

$$
u(t)=\left\{\begin{array}{l}
\frac{-E_{q}(-\lambda) E_{q}\left(-t^{q} \lambda\right)}{1+E_{q}(-\lambda)} \sum_{i=1}^{m} \frac{I_{i}\left(u\left(t_{i}\right)\right)}{E_{q}\left(-t_{i}^{q} \lambda\right)}+\int_{0}^{t}(t-s)^{q-1} E_{q, q}\left(-(t-s)^{q} \lambda\right) f(s, u(s), T u(s), S u(s)) d s \\
\quad-\frac{E_{q}\left(-t^{q} \lambda\right)}{1+E_{q}(-\lambda)} \int_{0}^{1}(1-s)^{q-1} E_{q, q}\left(-(1-s)^{q} \lambda\right) f(s, u(s), T u(s), \operatorname{Su}(s)) d s, \quad t \in J_{0}, \\
\frac{E_{q}\left(-t^{q} \lambda\right)}{1+E_{q}(-\lambda)}\left\{\sum_{i=1}^{m} \frac{I_{i}\left(u\left(t_{i}\right)\right)}{E_{q}\left(-t_{i}^{q} \lambda\right)}-\int_{0}^{1}(1-s)^{q-1} E_{q, q}\left(-(1-s)^{q} \lambda\right) f(s, u(s), T u(s), \operatorname{Su}(s)) d s\right\} \\
\quad-E_{q}\left(-t^{q} \lambda\right) \sum_{j=k+1}^{m} \frac{I_{j}\left(u\left(t_{j}\right)\right)}{E_{q}\left(-t_{j}^{q} \lambda\right)} \\
\quad+\int_{0}^{t}(t-s)^{q-1} E_{q, q}\left(-(t-s)^{q} \lambda\right) f(s, u(s), T u(s), S u(s)) d s, \\
\quad t \in J_{k}, k=1,2, \ldots, m-1, \\
\frac{E_{q}\left(-t^{q} \lambda\right)}{1+E_{q}(-\lambda)}\left\{\sum_{i=1}^{m} \frac{I_{i}\left(u\left(t_{i}\right)\right)}{E_{q}\left(-t_{i}^{q} \lambda\right)}-\int_{0}^{1}(1-s)^{q-1} E_{q, q}\left(-(1-s)^{q} \lambda\right) f(s, u(s), T u(s), \operatorname{Su}(s)) d s\right\} \\
\quad+\int_{0}^{t}(t-s)^{q-1} E_{q, q}\left(-(t-s)^{q} \lambda\right) f(s, u(s), T u(s), \operatorname{Su}(s)) d s, \quad t \in J_{m} .
\end{array}\right.
$$

\section{Main results}

Theorem 3.1 Assume that conditions $\left(\mathrm{H}_{1}\right)-\left(\mathrm{H}_{3}\right)$ hold:

$\left(\mathrm{H}_{1}\right)$ There exist $L_{i}(t) \in C(J,(0,+\infty))(i=1,2,3)$ such that

$$
\left|f\left(t, u_{1}, v_{1}, w_{1}\right)-f\left(t, u_{2}, v_{2}, w_{2}\right)\right| \leq L_{1}(t)\left|u_{1}-u_{2}\right|+L_{2}(t)\left|v_{1}-v_{2}\right|+L_{3}(t)\left|w_{1}-w_{2}\right|
$$

for all $t \in J$ and $u_{j}, v_{j}, w_{j} \in \mathbf{R}, j=1,2$.

$\left(\mathrm{H}_{2}\right)$ There exists a constant $L_{4}>0$ such that

$$
\left|I_{k}(u)-I_{k}(v)\right| \leq L_{4}|u-v|, \quad u, v \in \mathbf{R}, k=1,2, \ldots, m
$$


$\left(\mathrm{H}_{3}\right)$

$$
\chi=\frac{3}{\left|1+E_{q}(-\lambda)\right|}\left(\sum_{i=1}^{m} \frac{L_{4}}{\left|E_{q}\left(-t_{i}^{q} \lambda\right)\right|}+\frac{\left(\overline{L_{1}}+\overline{L_{2}} k_{0}+\overline{L_{3}} h_{0}\right)}{\Gamma(q+1)}\right)<1,
$$

where $\overline{L_{j}}=\max \left\{L_{j}(t): t \in J\right\}, j=1,2,3, k_{0}=\max \{|k(t, s)|:(t, s) \in D\}$, and $h_{0}=\max \{|h(t, s)|:$ $(t, s) \in J \times J\}$.

Then the boundary value problem (1.3) has a unique solution.

Proof Let $M=\sup \{|f(t, 0,0,0)|: t \in J\}, M^{\prime}=\max \left\{\left|I_{i}(0)\right|: i=1,2, \ldots, m\right\}$, and $B_{r}=\{u \in$ $\left.P C(J, \mathbf{R}):\|u\|_{P C} \leq r\right\}$, where

$$
r \geq \frac{\sum_{i=1}^{m} \frac{M^{\prime}}{\left|E_{q}\left(-t_{i}^{q} \lambda\right)\right|}+\frac{M}{\Gamma(q+1)}}{\frac{\left|1+E_{q}(-\lambda)\right|}{3}-\left[\sum_{i=1}^{m} \frac{L_{4}}{\left|E_{q}\left(-t_{i}^{q} \lambda\right)\right|}+\frac{\overline{L_{1}}+\overline{L_{2}} k_{0}+\overline{L_{3}} h_{0}}{\Gamma(q+1)}\right]} .
$$

Define the operator $F: B_{r} \rightarrow P C(J, \mathbf{R})$ by

$$
\begin{aligned}
F u(t)= & \frac{E_{q}\left(-t^{q} \lambda\right)}{1+E_{q}(-\lambda)}\left\{\sum_{i=1}^{m} \frac{I_{i}\left(u\left(t_{i}\right)\right)}{E_{q}\left(-t_{i}^{q} \lambda\right)}\right. \\
& \left.-\int_{0}^{1}(1-s)^{q-1} E_{q, q}\left(-(1-s)^{q} \lambda\right) f(s, u(s), T u(s), S u(s)) d s\right\} \\
& -E_{q}\left(-t^{q} \lambda\right) \sum_{j=k+1}^{m} \frac{I_{j}\left(u\left(t_{j}\right)\right)}{E_{q}\left(-t_{j}^{q} \lambda\right)} \\
& +\int_{0}^{t}(t-s)^{q-1} E_{q, q}\left(-(t-s)^{q} \lambda\right) f(s, u(s), T u(s), S u(s)) d s, \\
& t \in J_{k}, k=0,1,2, \ldots, m .
\end{aligned}
$$

First, we show that $F\left(B_{r}\right) \subset B_{r}$. For any $u \in B_{r}$ and $t \in J$, by Lemma 2.1 we have

$$
\begin{aligned}
|(F u)(t)| & \left|E_{q}\left(-t^{q} \lambda\right)\right| \mid \frac{1}{1+E_{q}(-\lambda)}\left\{\sum_{i=1}^{m} \frac{I_{i}\left(u\left(t_{i}\right)\right)}{E_{q}\left(-t_{i}^{q} \lambda\right)}\right. \\
& \left.-\int_{0}^{1}(1-s)^{q-1} E_{q, q}\left(-(1-s)^{q} \lambda\right) f(s, u(s), T u(s), S u(s)) d s\right\}-\sum_{j=k+1}^{m} \frac{I_{j}\left(u\left(t_{j}\right)\right)}{E_{q}\left(-t_{j}^{q} \lambda\right)} \mid \\
& +\left|\int_{0}^{t}(t-s)^{q-1} E_{q, q}\left(-(t-s)^{q} \lambda\right) f(s, u(s), T u(s), S u(s)) d s\right| \\
\leq & \frac{1}{\left|1+E_{q}(-\lambda)\right|}\left\{\sum_{i=1}^{m} \frac{\left|I_{i}\left(u\left(t_{i}\right)\right)\right|}{\left|E_{q}\left(-t_{i}^{q} \lambda\right)\right|}+\frac{1}{\Gamma(q)} \int_{0}^{1}(1-s)^{q-1}|f(s, u(s), T u(s), S u(s))| d s\right\} \\
& +\sum_{i=1}^{m} \frac{\left|I_{i}\left(u\left(t_{i}\right)\right)\right|}{\left|E_{q}\left(-t_{i}^{q} \lambda\right)\right|}+\frac{1}{\Gamma(q)} \int_{0}^{t}(t-s)^{q-1}|f(s, u(s), T u(s), S u(s))| d s \\
\leq & \frac{1+\left|1+E_{q}(-\lambda)\right|}{\left|1+E_{q}(-\lambda)\right|}\left\{\sum_{i=1}^{m} \frac{\left|I_{i}\left(u\left(t_{i}\right)\right)-I_{i}(0)\right|+M^{\prime}}{\left|E_{q}\left(-t_{i}^{q} \lambda\right)\right|}\right\}
\end{aligned}
$$




$$
\begin{aligned}
& +\frac{1}{\Gamma(q)\left|1+E_{q}(-\lambda)\right|} \int_{0}^{1}(1-s)^{q-1}|f(s, u(s), T u(s), S u(s))-f(s, 0,0,0)| d s \\
& +\frac{1}{\Gamma(q)\left|1+E_{q}(-\lambda)\right|} \int_{0}^{1}(1-s)^{q-1}|f(s, 0,0,0)| d s \\
& +\frac{1}{\Gamma(q)} \int_{0}^{t}(t-s)^{q-1}|f(s, u(s), T u(s), \operatorname{Su}(s))-f(s, 0,0,0)| d s \\
& +\frac{1}{\Gamma(q)} \int_{0}^{t}(t-s)^{q-1}|f(s, 0,0,0)| d s \\
& \leq \frac{3}{\left|1+E_{q}(-\lambda)\right|} \sum_{i=1}^{m} \frac{L_{4} r+M^{\prime}}{\left|E_{q}\left(-t_{i}^{q} \lambda\right)\right|}+\frac{M}{\Gamma(q+1)\left|1+E_{q}(-\lambda)\right|}+\frac{M}{\Gamma(q+1)} \\
& +\frac{1}{\Gamma(q)\left|1+E_{q}(-\lambda)\right|} \int_{0}^{1}(1-s)^{q-1}\left[L_{1}(s)|u(s)|+L_{2}(s)|T u(s)|+L_{3}(s)|\operatorname{Su}(s)|\right] d s \\
& +\frac{1}{\Gamma(q)} \int_{0}^{t}(t-s)^{q-1}\left[L_{1}(s)|u(s)|++L_{2}(s)|T u(s)|+L_{3}(s)|\operatorname{Su}(s)|\right] d s \\
& \leq \frac{3}{\left|1+E_{q}(-\lambda)\right|}\left\{\sum_{i=1}^{m} \frac{L_{4} r+M^{\prime}}{\left|E_{q}\left(-t_{i}^{q} \lambda\right)\right|}+\frac{M}{\Gamma(q+1)}\right\} \\
& +\frac{1}{\Gamma(q)\left|1+E_{q}(-\lambda)\right|} \int_{0}^{1}(1-s)^{q-1}\left(\overline{L_{1}} r+\overline{L_{2}} k_{0} r+\overline{L_{3}} h_{0} r\right) d s \\
& +\frac{1}{\Gamma(q)} \int_{0}^{t}(t-s)^{q-1}\left(\overline{L_{1}} r+\overline{L_{2}} k_{0} r+\overline{L_{3}} h_{0} r\right) d s \\
& \leq \frac{3}{\left|1+E_{q}(-\lambda)\right|}\left\{\sum_{i=1}^{m} \frac{M^{\prime}}{\left|E_{q}\left(-t_{i}^{q} \lambda\right)\right|}+\frac{M}{\Gamma(q+1)}\right. \\
& \left.+\left[\sum_{i=1}^{m} \frac{L_{4}}{\left|E_{q}\left(-t_{i}^{q} \lambda\right)\right|}+\frac{\overline{L_{1}}+\overline{L_{2}} k_{0}+\overline{L_{3}} h_{0}}{\Gamma(q+1)}\right] r\right\} \\
& \leq r \text {. }
\end{aligned}
$$

Hence $F\left(B_{r}\right) \subset B_{r}$.

Next, we show that the operator $F$ is a contraction mapping. For any $t \in J$ and $u, v \in B_{r}$, we obtain

$$
\begin{aligned}
|(F u)(t)-(F v)(t)| & \mid \frac{E_{q}\left(-t^{q} \lambda\right)}{1+E_{q}(-\lambda)}\left\{\sum_{i=1}^{m} \frac{I_{i}\left(u\left(t_{i}\right)\right)-I_{i}\left(v\left(t_{i}\right)\right)}{E_{q}\left(-t_{i}^{q} \lambda\right)}\right. \\
& \left.\quad-\int_{0}^{1}(1-s)^{q-1} E_{q, q}\left(-(1-s)^{q} \lambda\right)(f(s, u(s), T u(s), S u(s))-f(s, v(s), T v(s), S v(s))) d s\right\} \\
& -E_{q}\left(-t^{q} \lambda\right) \sum_{j=k+1}^{m} \frac{I_{j}\left(u\left(t_{j}\right)\right)-I_{j}\left(v\left(t_{j}\right)\right)}{E_{q}\left(-t_{j}^{q} \lambda\right)} \\
& +\int_{0}^{t}(t-s)^{q-1} E_{q, q}\left(-(t-s)^{q} \lambda\right)(f(s, u(s), T u(s), S u(s))-f(s, v(s), T v(s), S v(s))) d s \mid \\
\leq & \left(\frac{1}{1+\left|E_{q}(-\lambda)\right|}+1\right) \sum_{i=1}^{m} \frac{L_{4}\left|u\left(t_{i}\right)-v\left(t_{i}\right)\right|}{\left|E_{q}\left(-t_{i}^{q} \lambda\right)\right|}+\frac{1}{\Gamma(q)\left|1+E_{q}(-\lambda)\right|}
\end{aligned}
$$




$$
\begin{aligned}
& \cdot \int_{0}^{1}(1-s)^{q-1}\left\{L_{1}(s)|u(s)-v(s)|+L_{2}(s) \int_{0}^{s}|k(s, \tau)||u(\tau)-v(\tau)| d \tau\right. \\
& \left.+L_{3}(s) \int_{0}^{1}|h(s, \tau)||u(\tau)-v(\tau)| d \tau\right\} d s+\frac{1}{\Gamma(q)} \int_{0}^{t}(t-s)^{q-1}\left\{L_{1}(s)|u(s)-v(s)|\right. \\
& \left.+L_{2}(s) \int_{0}^{s}|k(s, \tau)||u(\tau)-v(\tau)| d \tau+L_{3}(s) \int_{0}^{1}|h(s, \tau)||u(\tau)-v(\tau)| d \tau\right\} d s \\
\leq & \frac{3}{\left|1+E_{q}(-\lambda)\right|} \sum_{i=1}^{m} \frac{L_{4}\|u-v\|_{P C}}{\left|E_{q}\left(-t_{i}^{q} \lambda\right)\right|}+\frac{1}{\Gamma(q)\left|1+E_{q}(-\lambda)\right|} \\
& \cdot \int_{0}^{1}(1-s)^{q-1}\left(\overline{L_{1}}\|u-v\|_{P C}+\overline{L_{2}} k_{0}\|u-v\|_{P C}+\overline{L_{3}} h_{0}\|u-v\|_{P C}\right) d s \\
& +\frac{1}{\Gamma(q)} \int_{0}^{t}(t-s)^{q-1}\left(\overline{L_{1}}\|u-v\|_{P C}+\overline{L_{2}} k_{0}\|u-v\|_{P C}+\overline{L_{3}} h_{0}\|u-v\|_{P C}\right) d s \\
\leq & \frac{3}{\left|1+E_{q}(-\lambda)\right|}\left(\sum_{i=1}^{m} \frac{L_{4}}{\left|E_{q}\left(-t_{i}^{q} \lambda\right)\right|}+\frac{\left(\overline{L_{1}}+\overline{L_{2}} k_{0}+\overline{L_{3}} h_{0}\right)}{\Gamma(q+1)}\right)\|u-v\|_{P C} \\
= & \chi\|u-v\|_{P C} .
\end{aligned}
$$

Thus $\|F u-F v\|_{P C} \leq \chi\|u-v\|_{P C}$. Then from the Banach contraction mapping principle it follows that problem (1.3) has a unique solution. This completes the proof.

Theorem 3.2 Assume that condition $\left(\mathrm{H}_{2}\right)$ and the following conditions $\left(\mathrm{H}_{4}\right)-\left(\mathrm{H}_{5}\right)$ hold:

$\left(\mathrm{H}_{4}\right)$ There exist a function $\mu \in L^{\frac{1}{\sigma}}(J,(0,+\infty))(0<\sigma<q<1)$ and a nondecreasing function $\bar{\omega} \in C([0, \infty),(0,+\infty))$ such that

$$
|f(t, u(t), T u(t), S u(t))| \leq \mu(t) \bar{\omega}\left(\|u\|_{P C}\right), \quad u \in P C(J, \mathbf{R}), t \in J .
$$

$\left(\mathrm{H}_{5}\right)$

$$
\frac{3}{\left|1+E_{q}(-\lambda)\right|}\left(\frac{\|\mu\|_{L^{\frac{1}{\sigma}}(J)}}{\Gamma(q)\left(\frac{q-\sigma}{1-\sigma}\right)^{1-\sigma}} \liminf _{r \rightarrow+\infty} \frac{\bar{\omega}(r)}{r}+\sum_{i=1}^{m} \frac{L_{4}}{\left|E_{q}\left(-t_{i}^{q} \lambda\right)\right|}\right)<1
$$

Then the boundary value problem (1.3) has at least one solution.

Proof For $r>0$, the set $B_{r}=\left\{u \in P C(J, \mathbf{R}):\|u\|_{P C} \leq r\right\}$ is a bounded closed convex set in $P C(J, \mathbf{R})$. Define the operators $P$ and $Q$ on $B_{r}$ as

$$
\begin{aligned}
(P u)(t)= & \int_{0}^{t}(t-s)^{q-1} E_{q, q}\left(-(t-s)^{q} \lambda\right) f(s, u(s), T u(s), S u(s)) d s \\
& -\frac{E_{q}\left(-t^{q} \lambda\right)}{1+E_{q}(-\lambda)} \int_{0}^{1}(1-s)^{q-1} E_{q, q}\left(-(1-s)^{q} \lambda\right) f(s, u(s), T u(s), S u(s)) d s, \\
(Q u)(t)= & \frac{E_{q}\left(-t^{q} \lambda\right)}{1+E_{q}(-\lambda)} \sum_{i=1}^{m} \frac{I_{i}\left(u\left(t_{i}\right)\right)}{E_{q}\left(-t_{i}^{q} \lambda\right)}-E_{q}\left(-t^{q} \lambda\right) \sum_{j=k+1}^{m} \frac{I_{j}\left(u\left(t_{j}\right)\right)}{E_{q}\left(-t_{j}^{q} \lambda\right)} .
\end{aligned}
$$


By $\left(\mathrm{H}_{4}\right)$ and the Hölder inequality, for any $u \in B_{r}$, we have

$$
\begin{aligned}
& \int_{0}^{t}\left|(t-s)^{q-1} f(s, u(s), T u(s), S u(s))\right| d s \\
& \quad \leq \int_{0}^{t}\left|(t-s)^{q-1} \mu(s) \bar{\omega}(r)\right| d s \\
& \quad \leq\left(\int_{0}^{t}(t-s)^{\frac{q-1}{1-\sigma}} d s\right)^{1-\sigma}\left(\int_{0}^{t}(\bar{\omega}(r) \mu(s))^{\frac{1}{\sigma}} d s\right)^{\sigma} \\
& \quad \leq \frac{t^{q-\sigma}}{\left(\frac{q-\sigma}{1-\sigma}\right)^{1-\sigma}} \bar{\omega}(r)\|\mu\|_{L^{\frac{1}{\sigma}}(J)} \\
& \quad \leq \frac{\|\mu\|_{L^{\frac{1}{\sigma}}(J)}}{\left(\frac{q-\sigma}{1-\sigma}\right)^{1-\sigma}} \bar{\omega}(r) .
\end{aligned}
$$

Similarly, we have

$$
\int_{0}^{1}\left|(1-s)^{q-1} f(s, u(s), T u(s), S u(s))\right| d s \leq \frac{\|\mu\|_{L^{\frac{1}{\sigma}}}(J)}{\left(\frac{q-\sigma}{1-\sigma}\right)^{1-\sigma}} \bar{\omega}(r) .
$$

Next, we show that there exists $r_{0}>0$ with $P u+Q v \in B_{r_{0}}$ for $u, v \in B_{r_{0}}$. If this were not true, then, for each $r>0$, there would exist $u_{r}, v_{r} \in B_{r}$ and $t_{r} \in J$ such that $\mid\left(P u_{r}\right)\left(t_{r}\right)+$ $\left(Q v_{r}\right)\left(t_{r}\right) \mid>r$. Assumption $\left(\mathrm{H}_{2}\right)$ implies $\left|I_{i}\left(u\left(t_{i}\right)\right)\right| \leq\left|I_{i}\left(u\left(t_{i}\right)\right)-I_{i}(0)\right|+\left|I_{i}(0)\right| \leq L_{4} r+M^{\prime}$. Hence

$$
\begin{aligned}
r< & \left|\left(P u_{r}\right)\left(t_{r}\right)+\left(Q v_{r}\right)\left(t_{r}\right)\right| \\
\leq & \frac{\|\mu\|_{\left.L^{\frac{1}{\sigma}}()\right)}}{\Gamma(q)\left|1+E_{q}(-\lambda)\right|\left(\frac{q-\sigma}{1-\sigma}\right)^{1-\sigma}} \bar{\omega}(r) \\
& +\frac{\|\mu\|_{L^{\frac{1}{\sigma}}(J)}}{\Gamma(q)\left(\frac{q-\sigma}{1-\sigma}\right)^{1-\sigma}} \bar{\omega}(r)+\frac{1}{\left|1+E_{q}(-\lambda)\right|} \sum_{i=1}^{m} \frac{L_{4} r+M^{\prime}}{\left|E_{q}\left(-t_{i}^{q} \lambda\right)\right|}+\sum_{i=1}^{m} \frac{L_{4} r+M^{\prime}}{\left|E_{q}\left(-t_{i}{ }^{q} \lambda\right)\right|} \\
= & \left(\frac{\|\mu\|_{L^{\frac{1}{\sigma}}()}}{\Gamma(q)\left(\frac{q^{-\sigma} \sigma}{1-\sigma}\right)^{1-\sigma}} \bar{\omega}(r)+\sum_{i=1}^{m} \frac{L_{4} r+M^{\prime}}{\left|E_{q}\left(-t_{i}^{q} \lambda\right)\right|}\right)\left(1+\frac{1}{\left|1+E_{q}(-\lambda)\right|}\right) \\
\leq & \frac{3}{\left|1+E_{q}(-\lambda)\right|}\left(\frac{\|\mu\|_{\left.L^{\frac{1}{\sigma}}()\right)}}{\Gamma(q)\left(\frac{q-\sigma}{1-\sigma}\right)^{1-\sigma}} \bar{\omega}(r)+\sum_{i=1}^{m} \frac{L_{4} r+M^{\prime}}{\left|E_{q}\left(-t_{i}^{q} \lambda\right)\right|}\right) .
\end{aligned}
$$

Dividing both sides by $r$ and taking the lower limit as $r \rightarrow+\infty$, we obtain

$$
1 \leq \frac{3}{\left|1+E_{q}(-\lambda)\right|}\left(\frac{\|\mu\|_{L^{\frac{1}{\sigma}}(J)}}{\Gamma(q)\left(\frac{q-\sigma}{1-\sigma}\right)^{1-\sigma}} \liminf _{r \rightarrow \infty} \frac{\bar{\omega}(r)}{r}+\sum_{i=1}^{m} \frac{L_{4}}{\left|E_{q}\left(-t_{i}^{q} \lambda\right)\right|}\right),
$$

which contradicts condition $\left(\mathrm{H}_{5}\right)$. Thus, there exists $r_{0}>0$ such that $P u+Q v \in B_{r_{0}}$ for all $u, v \in B_{r_{0}}$.

For all $t \in J$ and $u, v \in B_{r}$, we get

$$
\begin{aligned}
& |(Q u)(t)-(Q v)(t)| \\
& \quad \leq \frac{\left|E_{q}\left(-t^{q} \lambda\right)\right|}{\left|1+E_{q}(-\lambda)\right|} \sum_{i=1}^{m} \frac{\left|I_{i}\left(u\left(t_{i}\right)\right)-I_{i}\left(v\left(t_{i}\right)\right)\right|}{\left|E_{q}\left(-t_{i}^{q} \lambda\right)\right|}
\end{aligned}
$$




$$
\begin{aligned}
& +\left|E_{q}\left(-t^{q} \lambda\right)\right| \sum_{i=1}^{m} \frac{\left|I_{i}\left(u\left(t_{i}\right)\right)-I_{i}\left(v\left(t_{i}\right)\right)\right|}{\left|E_{q}\left(-t_{i}^{q} \lambda\right)\right|} \\
\leq & \sum_{i=1}^{m} \frac{\left|I_{i}\left(u\left(t_{i}\right)\right)-I_{i}\left(v\left(t_{i}\right)\right)\right|}{\left|E_{q}\left(-t_{i}^{q} \lambda\right)\right|}\left(1+\frac{1}{\left|1+E_{q}(-\lambda)\right|}\right) \\
\leq & \frac{3}{\left|1+E_{q}(-\lambda)\right|} \sum_{i=1}^{m} \frac{L_{4}\left|u\left(t_{i}\right)-v\left(t_{i}\right)\right|}{\left|E_{q}\left(-t_{i}^{q} \lambda\right)\right|} \\
\leq & \frac{3}{\left|1+E_{q}(-\lambda)\right|} \sum_{i=1}^{m} \frac{L_{4}\|u-v\|_{P C}}{\left|E_{q}\left(-t_{i}^{q} \lambda\right)\right|} .
\end{aligned}
$$

Let $\chi^{\prime}=\frac{3}{\left|1+E_{q}(-\lambda)\right|} \sum_{i=1}^{m} \frac{L_{4}}{\left|E_{q}\left(-t_{i}^{q} \lambda\right)\right|}$. From $\left(\mathrm{H}_{5}\right)$ we have $0<\chi^{\prime}<1$ and $\|Q u-Q v\|_{P C} \leq \chi^{\prime} \| u-$ $v \|_{P C}$, so $Q$ is a contraction mapping.

The continuity of $f$ implies that the operator $P$ is continuous. We now prove that $P$ is a compact operator. Following the procedure used in the first part of Theorem 3.1, it follows that $P\left(B_{r}\right)$ is uniformly bounded on $P C(J, \mathbf{R})$. We now show that $P\left(B_{r}\right)$ is equicontinuous on $J_{k}(k=1, \ldots, m)$. Let $\Omega=J \times B_{r} \times T B_{r} \times S B_{r}$ and $\bar{f}=\sup _{(t, u, T u, S u) \in \Omega}|f(t, u, T u, S u)|$. Then, for any $t_{k}<\tau_{2}<\tau_{1} \leq t_{k+1}$, we have

$$
\begin{aligned}
& \left|(P u)\left(\tau_{2}\right)-(P u)\left(\tau_{1}\right)\right| \\
& \leq \mid \int_{0}^{\tau_{2}}\left(\tau_{2}-s\right)^{q-1} E_{q, q}\left(-\left(\tau_{2}-s\right)^{q} \lambda\right) f(s, u(s), T u(s), S u(s)) d s \\
& -\int_{0}^{\tau_{1}}\left(\tau_{1}-s\right)^{q-1} E_{q, q}\left(-\left(\tau_{1}-s\right)^{q} \lambda\right) f(s, u(s), T u(s), S u(s)) d s \mid \\
& +\left|\frac{E_{q}\left(-\tau_{2}^{q} \lambda\right)-E_{q}\left(-\tau_{1}^{q} \lambda\right)}{1+E_{q}(-\lambda)} \int_{0}^{1}(1-s)^{q-1} E_{q, q}\left(-(1-s)^{q} \lambda\right) f(s, u(s), T u(s), S u(s)) d s\right| \\
& \leq \mid \int_{0}^{\tau_{2}}\left(\tau_{2}-s\right)^{q-1} E_{q, q}\left(-\left(\tau_{2}-s\right)^{q} \lambda\right) f(s, u(s), T u(s), S u(s)) d s \\
& -\int_{0}^{\tau_{2}}\left(\tau_{1}-s\right)^{q-1} E_{q, q}\left(-\left(\tau_{2}-s\right)^{q} \lambda\right) f(s, u(s), T u(s), S u(s)) d s \\
& +\int_{0}^{\tau_{2}}\left(\tau_{1}-s\right)^{q-1} E_{q, q}\left(-\left(\tau_{2}-s\right)^{q} \lambda\right) f(s, u(s), T u(s), S u(s)) d s \\
& -\int_{0}^{\tau_{2}}\left(\tau_{1}-s\right)^{q-1} E_{q, q}\left(-\left(\tau_{1}-s\right)^{q} \lambda\right) f(s, u(s), T u(s), S u(s)) d s \\
& -\int_{\tau_{2}}^{\tau_{1}}\left(\tau_{1}-s\right)^{q-1} E_{q, q}\left(-\left(\tau_{1}-s\right)^{q} \lambda\right) f(s, u(s), T u(s), S u(s)) d s \mid \\
& +\frac{\left|E_{q}\left(-\tau_{2}{ }^{q} \lambda\right)-E_{q}\left(-\tau_{1}^{q} \lambda\right)\right|}{\Gamma(q)\left|1+E_{q}(-\lambda)\right|} \int_{0}^{1}(1-s)^{q-1}|f(s, u(s), \operatorname{Tu}(s), \operatorname{Su}(s))| d s \\
& \leq \int_{0}^{\tau_{2}}\left|\left(\tau_{2}-s\right)^{q-1}-\left(\tau_{1}-s\right)^{q-1}\right|\left|E_{q, q}\left(-\left(\tau_{2}-s\right)^{q} \lambda\right)\right| \bar{f} d s \\
& +\int_{0}^{\tau_{2}}\left(\tau_{1}-s\right)^{q-1}\left|E_{q, q}\left(-\left(\tau_{2}-s\right)^{q} \lambda\right)-E_{q, q}\left(-\left(\tau_{1}-s\right)^{q} \lambda\right)\right| \bar{f} d s \\
& +\frac{\bar{f}}{\Gamma(q)}\left|\int_{\tau_{2}}^{\tau_{1}}\left(\tau_{1}-s\right)^{q-1} d s\right|+\frac{\left|E_{q}\left(-\tau_{2}^{q} \lambda\right)-E_{q}\left(-\tau_{1} q \lambda\right)\right| \bar{f}}{\Gamma(q+1)\left|1+E_{q}(-\lambda)\right|} \\
& \leq \frac{\bar{f}}{\Gamma(q)}\left|\int_{0}^{\tau_{2}}\left(\left(\tau_{2}-s\right)^{q-1}-\left(\tau_{1}-s\right)^{q-1}\right) d s\right|+\frac{\left(\tau_{1}-\tau_{2}\right)^{q} \bar{f}}{\Gamma(q+1)}+\frac{\left|E_{q}\left(-\tau_{2}{ }^{q} \lambda\right)-E_{q}\left(-\tau_{1}{ }^{q} \lambda\right)\right| \bar{f}}{\Gamma(q+1)\left|1+E_{q}(-\lambda)\right|}
\end{aligned}
$$




$$
\begin{aligned}
& +\bar{f} \int_{0}^{\tau_{2}}\left(\tau_{1}-s\right)^{q-1}\left|E_{q, q}\left(-\left(\tau_{2}-s\right)^{q} \lambda\right)-E_{q, q}\left(-\left(\tau_{1}-s\right)^{q} \lambda\right)\right| d s \\
\leq & \frac{\left(\tau_{1}-\tau_{2}\right)^{q}+\tau_{1}^{q}-\tau_{2}^{q}}{\Gamma(q+1)} \bar{f}+\frac{\left(\tau_{1}-\tau_{2}\right)^{q} \bar{f}}{\Gamma(q+1)}+\frac{\left|E_{q}\left(-\tau_{2}{ }^{q} \lambda\right)-E_{q}\left(-\tau_{1}{ }^{q} \lambda\right)\right| \bar{f}}{\Gamma(q+1)\left|1+E_{q}(-\lambda)\right|} \\
& +\bar{f} \int_{0}^{\tau_{2}}\left(\tau_{1}-s\right)^{q-1}\left|E_{q, q}\left(-\left(\tau_{2}-s\right)^{q} \lambda\right)-E_{q, q}\left(-\left(\tau_{1}-s\right)^{q} \lambda\right)\right| d s .
\end{aligned}
$$

By Lemma 2.1(2) we know that $E_{q, q}\left(-t^{q} \lambda\right)$ is continuous on $t \in J$, and thus $E_{q, q}\left(-t^{q} \lambda\right)$ is uniformly continuous on $t \in J$. Hence, for any $\varepsilon>0$, there is a sufficiently small $\delta>0$ such that, for $t_{1}, t_{2} \in J$ with $\left|t_{1}-t_{2}\right|<\delta$, we have

$$
\left|E_{q, q}\left(-t_{1}^{q} \lambda\right)-E_{q, q}\left(-t_{2}^{q} \lambda\right)\right|<\frac{\varepsilon}{\tau_{2}^{\frac{q}{2-q}}} .
$$

Let $\sigma_{1}=\frac{2-q}{2(1-q)}$ and $\sigma_{2}=\frac{2-q}{q}$. Then $\sigma_{1}>1, \sigma_{2}>1$, and $\frac{1}{\sigma_{1}}+\frac{1}{\sigma_{2}}=1$. By the Hölder inequality we have

$$
\begin{aligned}
& \int_{0}^{\tau_{2}}\left(\tau_{1}-s\right)^{q-1}\left|E_{q, q}\left(-\left(\tau_{2}-s\right)^{q} \lambda\right)-E_{q, q}\left(-\left(\tau_{1}-s\right)^{q} \lambda\right)\right| d s \\
& \leq {\left[\int_{0}^{\tau_{2}}\left(\tau_{1}-s\right)^{(q-1) \frac{2-q}{2(1-q)}} d s\right]^{\frac{2(1-q)}{2-q}} } \\
& \cdot\left[\int_{0}^{\tau_{2}}\left(E_{q, q}\left(-\left(\tau_{2}-s\right)^{q} \lambda\right)-E_{q, q}\left(-\left(\tau_{1}-s\right)^{q} \lambda\right)\right)^{\frac{2-q}{q}} d s\right]^{\frac{q}{2-q}} \\
& \leq {\left[\frac{\tau_{1}^{\frac{q}{2}}-\left(\tau_{1}-\tau_{2}\right)^{\frac{q}{2}}}{\frac{q}{2}}\right]^{\frac{2(1-q)}{2-q}} \cdot\left[\int_{0}^{\tau_{2}}\left(\frac{\varepsilon}{\tau_{2}^{\frac{q}{2-q}}}\right)^{\frac{2-q}{q}} d s\right]^{\frac{q}{2-q}} } \\
&= {\left[\frac{2 \tau_{1}^{\frac{q}{2}}-2\left(\tau_{1}-\tau_{2}\right)^{\frac{q}{2}}}{q}\right]^{\frac{2(1-q)}{2-q}} \cdot \varepsilon, }
\end{aligned}
$$

so $\int_{0}^{\tau_{2}}\left(\tau_{1}-s\right)^{q-1}\left|E_{q, q}\left(-\left(\tau_{2}-s\right)^{q} \lambda\right)-E_{q, q}\left(-\left(\tau_{1}-s\right)^{q} \lambda\right)\right| d s$ tends to zero as $\tau_{2} \rightarrow \tau_{1}$. Therefore, $\left|(P u)\left(\tau_{2}\right)-(P u)\left(\tau_{1}\right)\right|$ tends to zero as $\tau_{2} \rightarrow \tau_{1}$. This yields that $P$ is equicontinuous on the interval $J_{k}$.

Combining the above arguments and the $P C$-type Arzelà-Ascoli theorem (Lemma 2.3 in the case $X=\mathbf{R}$ ), we conclude that $P: B_{r} \rightarrow B_{r}$ is compact and completely continuous. Then it follows from Lemma 2.2 that problem (1.3) has at least one solution. This completes the proof.

\section{Examples}

In this section, we give two examples to illustrate our main results.

Example 4.1 Consider the following impulsive fractional integro-differential equation with antiperiodic boundary condition:

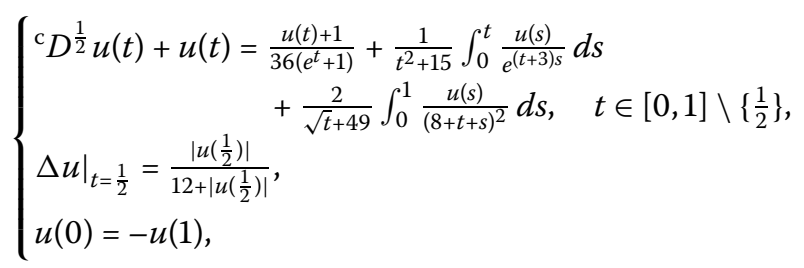


Let

$$
\begin{aligned}
& f(t, u, v, w)=\frac{u+1}{36\left(e^{t}+1\right)}+\frac{v}{t^{2}+15}+\frac{2 w}{\sqrt{t}+49}, \quad I_{k}(u)=\frac{|u|}{12+|u|}, \\
& (T u)(t)=\int_{0}^{t} e^{-(t+3) s} u(s) d s, \quad(S u)(t)=\int_{0}^{1} \frac{u(s)}{(8+t+s)^{2}} d s .
\end{aligned}
$$

By direct computation, $k_{0}=\max \left\{\frac{1}{e^{(t+3) s}}: 0 \leq s \leq t \leq 1\right\}=1$ and $h_{0}=\max \left\{\frac{1}{(8+t+s)^{2}}: 0 \leq s, t \leq\right.$ $1\}=\frac{1}{64}$. For $u_{1}, u_{2}, v_{1}, v_{2}, \omega_{1}, \omega_{2} \in \mathbf{R}$ and $t \in J$, we have

$$
\begin{aligned}
& \left|f\left(t, u_{1}, v_{1}, w_{1}\right)-f\left(t, u_{2}, v_{2}, w_{2}\right)\right| \\
& \quad \leq \frac{1}{36\left(e^{t}+1\right)}\left|u_{1}-u_{2}\right|+\frac{1}{t^{2}+15}\left|v_{1}-v_{2}\right|+\frac{2}{\sqrt{t}+49}\left|w_{1}-w_{2}\right|, \\
& \left|I_{k}\left(u_{1}\right)-I_{k}\left(u_{2}\right)\right| \leq \frac{1}{12}\left|u_{1}-u_{2}\right| .
\end{aligned}
$$

Let

$$
\begin{array}{ll}
L_{1}(t)=\frac{1}{36\left(e^{t}+1\right)}, & L_{2}(t)=\frac{1}{t^{2}+15}, \\
L_{3}(t)=\frac{2}{\sqrt{t}+49}, & L_{4}=\frac{1}{12} .
\end{array}
$$

It is easy to see that $\overline{L_{1}}=\frac{1}{72}, \overline{L_{2}}=\frac{1}{15}, \overline{L_{3}}=\frac{2}{49}, E_{\frac{1}{2}}(-1)=\frac{1+\frac{\pi-2}{\sqrt{\pi}}}{1+\sqrt{\pi+(\pi-2)}} \approx 0.42, E_{\frac{1}{2}}\left(-\left(\frac{1}{2}\right)^{\frac{1}{2}}\right) \approx 0.52$, $\Gamma\left(\frac{3}{2}\right)=\frac{1}{2} \sqrt{\pi} \approx 0.89$,

$$
\begin{aligned}
\chi & =\frac{3}{\left|1+E_{\frac{1}{2}}(-1)\right|}\left(\frac{L_{4}}{\left|E_{\frac{1}{2}}\left(-\left(\frac{1}{2}\right)^{\frac{1}{2}}\right)\right|}+\frac{\left(\overline{L_{1}}+\overline{L_{2}} k_{0}+\overline{L_{3}} h_{0}\right)}{\Gamma\left(\frac{3}{2}\right)}\right) \\
& \approx \frac{3}{1+0.42}\left(\frac{\frac{1}{12}}{0.52}+\frac{\frac{1}{72}+\frac{1}{15}+\frac{2}{49} \times \frac{1}{64}}{0.89}\right)<1 .
\end{aligned}
$$

Then by Theorem 3.1 problem (4.1) has a unique solution.

Example 4.2 Consider the following impulsive antiperiodic problem:

$$
\left\{\begin{aligned}
&{ }^{\mathrm{c}} D^{\frac{1}{2}} u(t)+u(t)=\left(\frac{\sqrt[3]{t+1}}{16}+\frac{1}{16 \sqrt[3]{t+1}}\right) \frac{|u(t)|}{1+|u(t)|}+\frac{\sqrt[3]{t+1}}{16 e^{t}} \sin \left(\int_{0}^{t} \sin (t-s) u(s) d s\right) \\
&+\frac{1}{16 \sqrt[3]{t+1}} \cos \left(\int_{0}^{1} \frac{u(s)}{1+t s} d s\right), \quad t \in[0,1] \backslash\left\{\frac{1}{2}\right\}, \\
&\left.\Delta u\right|_{t=\frac{1}{2}}=\frac{\left|u\left(\frac{1}{2}\right)\right|}{12+\left|u\left(\frac{1}{2}\right)\right|} \\
& u(0)=-u(1),
\end{aligned}\right.
$$

where

$$
f(t, u, v, w)=\left(\frac{\sqrt[3]{t+1}}{16}+\frac{1}{16 \sqrt[3]{t+1}}\right) \frac{|u|}{1+|u|}+\frac{\sqrt[3]{t+1}}{16 e^{t}} \sin v+\frac{1}{16 \sqrt[3]{t+1}} \cos w
$$


By computation we obtain

$$
\begin{aligned}
|f(t, u, T u, S u)| & \leq \frac{\sqrt[3]{t+1}}{16}+\frac{1}{16 \sqrt[3]{t+1}}+\frac{\sqrt[3]{t+1}}{16}\|u\|_{P C}+\frac{1}{16 \sqrt[3]{t+1}}\|u\|_{P C} \\
& =\left(\frac{\sqrt[3]{t+1}}{16}+\frac{1}{16 \sqrt[3]{t+1}}\right)\left(\|u\|_{P C}+1\right) .
\end{aligned}
$$

Let $\mu(t)=\frac{\sqrt[3]{t+1}}{16}+\frac{1}{16 \sqrt[3]{t+1}}, \sigma=\frac{1}{3}$, and $\bar{\omega}(r)=r+1$. Then $\liminf _{r \rightarrow \infty} \frac{\bar{\omega}(r)}{r}=1$ and $L_{4}=\frac{1}{12}$. Thus,

$$
\left\{\frac{\frac{1}{12}}{E_{\frac{1}{2}}\left(-\left(\frac{1}{2}\right)^{\frac{1}{2}}\right)}+\frac{\left[\int_{0}^{1}\left(\frac{\sqrt[3]{t+1}}{16}+\frac{1}{16 \sqrt[3]{t+1}}\right)^{3} d t\right]^{\frac{1}{3}}}{\Gamma\left(\frac{1}{2}\right)\left(\frac{\frac{1}{2}-\frac{1}{3}}{1-\frac{1}{3}}\right)^{1-\frac{1}{3}}}\right\} \frac{3}{1+E_{\frac{1}{2}}(-1)} \approx 0.95<1
$$

By Theorem 3.2 problem (4.2) has at least one solution.

\section{Conclusion}

In this paper, we are concerned with the existence and uniqueness of solutions for impulsive fractional integro-differential equation of mixed type with constant coefficient and antiperiodic boundary condition. The paper has several new features. First, we consider the impulsive fractional integro-differential equation of mixed type, that is, the nonlinear $f$ involves linear operators $T$ and $S$. The second new feature is that we studied antiperiodic boundary value problems with constant coefficients. Our results are based on the Banach contraction mapping principle and the Krasnoselskii fixed point theorem.

\section{Acknowledgements}

The authors would like to thank the referees for their pertinent comments and valuable suggestions.

\section{Funding}

This work is supported financially by the National Natural Science Foundation of China $(11501318,11371221)$, the Natural Science Foundation of Shandong Province of China (ZR2015AM022, ZR2014AM032), and the China Postdoctoral Science Foundation (2017M612230).

\section{Abbreviations}

Not applicable.

\section{Availability of data and materials}

Not applicable.

\section{Competing interests}

The authors declare that they have no competing interests.

Authors' contributions

All authors contributed equally to the writing of this paper. The authors read and approved the final manuscript.

\section{Author details}

${ }^{1}$ School of Mathematical Sciences, Qufu Normal University, Qufu, Shandong 273165, P.R. China. ${ }^{2}$ Department of Mathematics and Statistics, Curtin University, Perth, WA 6845, Australia. ${ }^{3}$ Department of Applied Mathematics, Shandong University of Science and Technology, Qingdao, 266590, P.R. China.

\section{Publisher's Note}

Springer Nature remains neutral with regard to jurisdictional claims in published maps and institutional affiliations. 


\section{References}

1. Kilbas, AA, Srivastava, HM, Trujillo, JJ: Theory and Applications of Fractional Differential Equations. Elsevier, Amsterdam (2006)

2. Podlubny, I: Fractional Differential Equations. Academic Press, New York (1999)

3. Baleanu, D, Diethelm, K, Scalas, E, Trujillo, JJ: Fractional Calculus Models and Numerical Methods. World Scientific, Boston (2012)

4. Wang, Y, Liu, L: Uniqueness and existence of positive solutions for the fractional integro-differential equation. Bound. Value Probl. 2017, 13 (2017)

5. Wang, Y, Liu, L: Positive solutions for a class of fractional 3-point boundary value problems at resonance. Adv. Differ. Equ. 2017, 7 (2017)

6. Cui, Y: Uniqueness of solution for boundary value problems for fractional differential equations. Appl. Math. Lett. 51, 48-54 (2016)

7. Zhang, $X, L i u, L, W u, Y$ : The uniqueness of positive solution for a fractional order model of turbulent flow in a porous medium. Appl. Math. Lett. 37, 26-33 (2014)

8. Zhang, X, Liu, L, Wu, Y: The iterative solutions of nonlinear fractional differential equations. Appl. Math. Comput. 219, 4680-4691 (2013)

9. Bai, Z, Zhang, S, Sun, S, Yin, C: Monotone iterative method for fractional differential equations. Electron. J. Differ. Equ. 2016, 6 (2016)

10. Bai, Z, Chen, Y, Lian, H, Sun, S: On the existence of blow up solutions for a class of fractional differential equations. Fract. Calc. Appl. Anal. 17, 1175-1187 (2014)

11. Zou, Y, Cui, Y: Existence results for a functional boundary value problem of fractional differential equations. Adv. Differ Equ. 2013, 233 (2013)

12. Cui, Y: Existence results for singular boundary value problem of nonlinear fractional differential equation. Abstr. Appl. Anal. 2011, Article ID 605614 (2011)

13. Hao, X: Positive solution for singular fractional differential equations involving derivatives. Adv. Differ. Equ. 2016, 139 (2016)

14. Hao, X, Liu, L, Wu, Y: Positive solutions for nonlinear fractional semipositone differential equation with nonlocal boundary conditions. J. Nonlinear Sci. Appl. 9, 3992-4002 (2016)

15. Liu, X, Jia, M: Existence of solutions for the integral boundary value problems of fractional order impulsive differential equations. Math. Methods Appl. Sci. 39, 475-487 (2016)

16. Agarwal, RP, Ahmad, B, Nieto, JJ: Fractional differential equations with nonlocal (parametric type) anti-periodic boundary conditions. Filomat 31, 1207-1214 (2017)

17. Mahmudov, N, Unul, S: On existence of BVP's for impulsive fractional differential equations. Adv. Differ. Equ. 2017، 15 (2017)

18. Wang, J, Feckan, M, Zhou, Y: A survey on impulsive fractional differential equations. Fract. Calc. Appl. Anal. 19, 806-831 (2016)

19. Liu, Y: Survey and new results on boundary-value problems of singular fractional differential equations with impulse effects. Electron. J. Differ. Equ. 2016, 296 (2016)

20. Liu, Y: Boundary value problems of singular multi-term fractional differential equations with impulse effects. Math. Nachr. 289, 1526-1547 (2016)

21. Bai, Z, Dong, X, Yin, C: Existence results for impulsive nonlinear fractional differential equation with mixed boundary conditions. Bound. Value Probl. 2016, 63 (2016)

22. $\mathrm{Xu}, \mathrm{L}, \mathrm{Hu}, \mathrm{H}$, Qin, F: Ultimate boundedness of impulsive fractional differential equations. Appl. Math. Lett. 62, 110-117 (2016)

23. Bonanno, G, Rodriguez-Lopez, R, Tersian, S: Existence of solutions to boundary value problem for impulsive fractional differential equations. Fract. Calc. Appl. Anal. 17, 717-744 (2014)

24. Rehman, MU, Eloe, PW: Existence and uniqueness of solutions for impulsive fractional differential equations. Appl. Math. Comput. 224, 422-431 (2013)

25. Liu, Z, Li, X: Existence and uniqueness of solutions for the nonlinear impulsive fractional differential equations. Commun. Nonlinear Sci. Numer. Simul. 18, 1362-1373 (2013)

26. $\mathrm{Xu}, \mathrm{Y}, \mathrm{Liu}, \mathrm{X}$ : Some boundary value problems of fractional differential equations with fractional impulsive conditions. J. Comput. Anal. Appl. 19, 426-443 (2015)

27. Nyamoradi, N, Rodriguez-Lopez, R: On boundary value problems for impulsive fractional differential equations. Appl. Math. Comput. 271, 874-892 (2015)

28. Yang, S, Zhang, S: Impulsive boundary value problem for a fractional differential equation. Bound. Value Probl. 2016 $203(2016)$

29. Shu, X, Shi, Y: A study on the mild solution of impulsive fractional evolution equations. Appl. Math. Comput. 273, 465-476 (2016)

30. Li, B, Gou, H: Existence of solutions for impulsive fractional evolution equations with periodic boundary condition Adv. Differ. Equ. 2017, 236 (2017)

31. Wang, G, Ahmad, B, Zhang, L, Nieto, JJ: Comments on the concept of existence of solution for impulsive fractiona differential equations. Commun. Nonlinear Sci. Numer. Simul. 19, 401-403 (2014)

32. Feckan, M, Wang, J, Zhou, Y: Response to 'Comments on the concept of existence of solution for impulsive fractional differential equations.' Commun. Nonlinear Sci. Numer. Simul. 19, 4213-4215 (2014)

33. Wang, G, Ahmad, B, Zhang, L: Impulsive anti-periodic boundary value problem for nonlinear differential equations of fractional order. Nonlinear Anal. 74, 792-804 (2011)

34. Li, X, Chen, F, Li, X: Generalized anti-periodic boundary value problems of impulsive fractional differential equations. Commun. Nonlinear Sci. Numer. Simul. 18, 28-41 (2013)

35. Liu, X, Li, Y: Some antiperiodic boundary value problem for nonlinear fractional impulsive differential equations. Abstr. Appl. Anal. 2014, Article ID 571536 (2014)

36. Ahmad, B, Nieto, JJ: Existence of solution for impulsive anti-periodic boundary value problems of fractional order. Taiwan. J. Math. 15, 981-993 (2011) 
37. Zhang, L, Wang, G: Existence of solutions for nonlinear fractional differential equations with impulses and anti-periodic boundary conditions. Electron. J. Qual. Theory Differ. Equ. 2011, 7 (2011)

38. Chen, $A$, Chen, Y: Existence of solutions to anti-periodic boundary value problem for nonlinear fractional differential equations with impulses. Adv. Differ. Equ. 2011, Article ID 915689 (2011)

39. Benchohra, M, Slimani, BA: Existence and uniqueness of solutions to impulsive fractional differential equations. Electron. J. Differ. Equ. 2009, 10 (2009)

40. Wang, J, Lin, Z: On the impulsive fractional anti-periodic BVP modelling with constant coefficients. J. Appl. Math. Comput. 46, 107-121 (2014)

41. Wang, J, Feckan, M, Zhou, Y: Presentation of solutions of impulsive fractional Langevin equations and existence results. Eur. Phys. J. Spec. Top. 222, 1857-1874 (2013)

42. Kransnoselskii, MA: Two remarks on the method of successive approximations. Usp. Mat. Nauk 10, 123-127 (1955)

43. Wei, W, Xiang, X, Peng, Y: Nonlinear impulsive integro-differential equations of mixed type ang optimal controls. Optimization 55, 141-156 (2006)

\section{Submit your manuscript to a SpringerOpen ${ }^{\circ}$} journal and benefit from:

- Convenient online submission

- Rigorous peer review

- Open access: articles freely available online

- High visibility within the field

- Retaining the copyright to your article

Submit your next manuscript at $\gg$ springeropen.com 